\title{
The Influence of Dietary Restrictions and Malnutrition on Morphological Construction of Bone Marrow
}

\author{
N. Kozak $^{1 *}$, M.Krośniak ${ }^{1}$ \\ ${ }^{1}$ Department of Food Chemistry and Nutrition, Jagiellonian University Medical College, 9 Medyczna Str, 30-688 \\ Cracow, Poland
}

\begin{abstract}
Well-balanced diet impacts on nutritional status. It is an important factor which determines proper forming of haematopoietic cells of bone marrow. Deficit as well as excess of diet components or calories can disturb functions of bone marrow and cause development of many diseases, including anaemia. The review of actual knowledge on the influence of dietary restrictions and malnutrition on functions of haematopoietic stem cells and erythrogenesis has been done in this paper. It was conducted many researches related to changes in animal's peripheral blood with limited caloric intake, but only some of them referred to the influence of malnutrition on bone marrow haematopoiesis. Malnutrition and commonly tested lack of protein in diet can cause hypoplasia and impaired immune function. Moderate caloric restrictions which do not cause malnutrition can contribute to slow down in ageing and to prevent chronic diseases. The composition of diet and its influence on morphological construction of bone marrow is little known issue with high innovative potential. In view of wide usage of animal model in assessment of drug safety, it should be considered to explore this knowledge and conduct additional researches.
\end{abstract}

Keywords: Bone marrow, Hematopoiesis, Malnutrition, Dietary restrictions

\section{Introduction}

A diet and good nutrition are very important elements which impact on proper functioning of organism. There are risk factors of many chronic diseases relatively easy to modify such as obesity, cardiovascular diseases, diabetes or osteoporosis. Deficit and excess of component taken with food can affect nutritional status. The symptoms can often concern whole organism or particular organ or structure.

The proper diet has huge importance for right formation of haematopoietic cells of bone marrow. Because of intensive divisions and maturation of cells, bone marrow is a structure which is very vulnerable to damaging agents activity. The damaging activity on bone marrow can manifest in extreme case as aplasia - entire decline of haematopoietic tissue which yield inhibition of morphotic blood elements, or hypoplasia - partial damage of haematopoietic tissue. It has been long since known that the lack of good quality proteins, ferrum and energy value in diet impact on erythrogenesis $[1,5,10]$. There are also other agents which impair bone marrow such as gamma rays, cobalt, benzene and arsenic (III) compounds.
However, the influence of particular diet stillstays the open issue which is worth devoting attention of researchers. This kind of researches have also high innovative potential with ability to make of the results in prevention of some diseases.

The influence of malnutrition on haematopoiesis and morphological construction of bone marrow The long term malnutrition of organism leads i.a. to disfunction of physiological and biochemical processes which concern also erythropoiesis. Significant role in haematopoiesis process have vitamins: C, B12, B6, folic acid and mineral components such as $\mathrm{Fe}, \mathrm{Cu}$ and

Corresponding Author: Natalia Kozak, Department of Food Chemistry and Nutrition, Jagiellonian University Medical College, Poland E-mail: nataliakozak90@gmail.com Received date: March 08, 2019; Accepted date: March 15, 2019; Published date: March 18, 2019.

DOI: https://doi.org/10.31546/IJFSNR.1001 
Co. In researches conducted on rats it was proved that nutritional status has vital influence on erythropoiesis. In Asanuma et al. (2011) 5 groups of animals where observed. Two of them had reduced amount of food by $33 \%$ or $66 \%$ for 14 days. Two next groups were subject to serially repeated phlebotomy together with reduced amount of food $(33 \%$ or $66 \%)$ during which they were bled (1\% or $4 \%$ total blood volume). The procedure was also done for 14 days. The fifth group was a control group, without any dietary restrictions and phlebotomy. It is worth pointing out that rats which were bled, characterised by statistically lower food intake than control group. Then the research leads up to comparison of groups of drugs which lost some volume of blood and were undernourished with groups of animals which were only undernourished. The phlebotomy was applied as an additionally erythrocyte forming stimulating factor so that the activity of confounding factors on the study could be excluded. The influence of bleeding on smaller food intake and therefore smaller weight gain was observed also in Kurata et al. (1997) research. The results of discussed research indicate the reduction of amount of reticulocyte in peripheral blood in group of rats with reduced food intake and increased bone marrow indicator M:E (myeloid line to erythroid line in bone marrow). The opposite conclusions have been drawn on the grounds of the group of rats with phlebotomy where increase of the number of reticulocytes was showed. It is proved by Furuhama et al. The measurement of reticulocytes in peripheral blood is necessary to assess erythropoiesis because the number of reticulocytes reflects erythropoetic activity of bonne marrow. The research shows that despite of inhibition agents activity (malnutrition) there is the ability to erythropoiesis in response to increased demand of organism (phlebotomy) but to a limited extent.

The research described above seems to be the continuation of Ogawa et al. (1985) - research in which rats were under similar caloric restrictions $(33 \%, 66 \%)$ for 3 months. Results of the research also indicate significant decrease of the number of reticulocytes in rat's peripheral blood with limited access to food. Moreover clear decrease in amount of progenitor cells of the line of red blood cells NRBC (erythroblast) in bone marrow was observed. It testifies the inhibition of erythropoiesis. To similar conclusions came earlier Dunn (1980) [7]. In researches beside negative energy balance the level of hydration was taken into account. The results of the research indicate the suppression of erythropoiesis by decreasing the vulnerability of target cell in spleen to erythropoietin (EPO), caused by decreased food intake. This effect was enhanced by dehydration. The author pointed out the meaning of the maintenance of proper caloric and water-electrolyte balance in regulation of erythropoiesis. The influence of diet restriction on erythroid line, progenitor cell proliferation was tested also by Hishinuma et al. $1990[10,12]$.

Mice with limited food supply had increased level of erythropoietin (EPO) which stimulates erythropoiesis. Increased in that amount of erythroid stem cell (CFUS) was associated with appearance of big amount of microcytes, without enhanced synthesis of haemoglobin. Anaemia was not observed. It was explained by intensified erythropoiesis. Presented research was an attempt to explain previous this author's observations (1989). It was stated then that there is increased production of microcytes and shortliving erythrocytes. It was caused by $40 \%$ limitation of food in mouse diet, without occurrence of anaemia.

There is a research that analyses the influence of different caloric restrictions on toxicological parameters, i.a. morphological construction of bone marrow. This was conducted by Moriyama et al. 2008 [20]. In this research 3 groups of rats were counted. They were given $75 \%, 50 \%$ and $25 \%$ of food in proportion to control group. The optimal amount of food for control group was established on the grounds of previous researches. Changes observed after 2 weeks in particular groups of animals were determined as very light $(+)$, light $(++)$, medium $(+++)$, major $(++++)$ and severe $(+++++)$. Based on the results of described researches the level of depletion of bone marrow was expressed. The depletion level was successfully increasing together with the growth of food restrictions. Decrease in amount of myeloid and erythroid line cells was set down.The same caloric restrictions were used earlier by Levin et al. (1993) in his research. The rats got relatively $75 \%$ (small restriction), $50 \%$ (moderate restriction) and $25 \%$ (major restriction) the amount of feed taken by control group [17]. This was haemoconcentration (blood concentration) observed in every group. It was involved directly with the level of limitation of feed what might be caused by mild dehydration of animals. The most important biological changes referred to the bone marrow and thymus [21].

Groups with mild and moderate restriction of feed were characterised by progressive inhibition of haematopoiesis in bone marrow. Along with decreased number of stem cells in bone marrow, the quantity of fat was increasing. In view of decreased fat tissue level caused by malnutrition at animals we can assume that bone marrow is a preferable place for gathering fat. The fat in bone marrow creates probably appropriate biochemical and nutritional environment for remaining cells [24]. 
The influence of malnutrition and 5-fluorouracil supply on haematopoiesis and morphological construction of bone marrow

Vast majority of available researches are primarily focused on the meaning of malnutrition concerning erythropoiesis. Miyata et al. (2009) analysed also other haematopoietic pathways, that is granulopoiesis and lymphocytopoiesis. There were 7 groups of male rats in the research: 3 of them were put to food limitations, next 3 apart from food restrictions additionally were taking 5-fluorouracil, cytostatic which damages bone marrow. The last group was the control group. Due to 5-FU it was possible to distinguish the symptoms of administration of the medicine and the consequences of food limitation. All groups were under observation for 21 days. Groups of rats with food restrictions got the same amount of feed for whole the time of experiment. Groups with cytostatic, took it for 14 days, after that was week break for recovery necessary to reverse changes. Results of experiment shows that majority of changes in haematopoietic system came simultaneously in every examined group. Decrease in the number of erythroblasts and increase of marrow indicator $\mathrm{M}: \mathrm{E}$ were observed. It indicates impairment of erythropoiesis. Decrease of progenitors, lymphocytes and granulocytes was also showed. Changes resulted from taking 5-FU by animals concerned the most probably only appearance of abnormal, polyploidal granulocytes in bone marrow $(<1 \%)$. Researchers suggest that inhibition activity of caloric deficit on haematopoiesis is crucial and it should be taken into account especially during checking hematotoxicity of drugs. The authors of mentioned research (Fumiko et al., 2010) decided to repeat the experiment this time minimizing changes caused by duration and shortened it to 4 days. Observed changes were supposed to reflect direct influence of 5-FU on haematopoiesis. After finishing the observation this was decrease in number of reticulocytes in peripheral blood and erythroid cells in bone marrow in rats which had 5-FU administered. In group of animals which had only food limitation this kind of effect were not observed. In relation to exception of secondary changes involved with malnutrition during 4-days research, it was admitted that 5-FU has direct influence on erythroblastic cells. Results prove that there is need to plan researches in adequate time frame. It is due to decrease maximally appearance of secondary changes involved with malnutrition and to assess correctly changes on the level of erythroblastic cells as an answer to medicine activity[18,19,20].

One more experiment was carried out (Asanuma et al., 2009) this time it was to check whether adult rats (12 weeks) with decreased body weight might be used to assessment of myelotoxicity. The results of the study were compared with data obtained from previous research, where young animals (6 weeks) were observed. In experiments due to big availability 6-weeks rats are mainly used. In subacute, chronic and neoplastic diseases in order to assess safety of the new drug its administrating in different periods of life is necessary. Moreover young animals in fast grow period might be more vulnerable to histopathological changes under malnutrition.

Similarly to previous researches 7 groups of animals were under analysis: control group, 3 groups with dietary restrictions and 3 groups which had limited feed and 5-fluorouracil given. The results of experiment indicate differences in lymphoid and granulocytic line depending on the age. Decrease of the number of white blood cells in peripheral blood and inhibition of haematopoiesis was more clear in young animals. There were no such significant differences between malnourished group and group which took 5FU additionally. This is indicative of big vulnerability of haematopoiesis to changes, especially among young animals which bone marrow cells are in constant increased proliferation and differentiation. It can be assumed that adult rats with decreased body weight caused by taking compound or medicine will be more relevant to assess their myelotoxicity $[2,3]$.

\section{The influence of caloric restrictions without causing malnutrition on ageing process of phenotype of stem cells}

Tang et al. (2016) have carried out an interesting and innovative research in which consequences of $30 \%$ food restriction among adult animals were analysed. It included influence on functions of haematopoietic stem cell (HSC) [23]. It was assumed that used reduction will not cause malnutrition but improve health state and delay tissue ageing. It should be underline that there are a lot of studies which describe changes in stem cells and dynamics of proliferation of haematopoietic cells depending on the age. However the level of nutrition was not taken into account.

As it was shown in described research, caloric restrictions delayed ageing process of phenotype of stem cells. Decrease of stem cells in resting state (not entering cell cycle) and improvement of ability to repopulation of HSC during ageing was also observed. Apart from described positive effects of dietary restrictions adverse effects were seen. The proliferation of lymphocyte stem cells was stopped what caused smaller production of lymphocytes. This leaded to impairment of immune functions. 
These researches are necessary because positive influence of moderate limitation of food on live length and psychomotoric efficiency was earlier observed in yeasts and rodents (Fontana et al., 2010) [9].

In different study analysing the influence of caloric restrictions without malnutrition on slowdown of ageing process VSEL (very small embryonic-like stem cell) were taken into account. VSEL are small bone marrow cells with embryonal characteristics. As it was showed VSEL can be activated and mobilized to peripheral blood. In adequate animal models they can contribute to regeneration of organs. They can also take part in maintenance of the stem cell pool. Their number correlate with length of life of tested mice. The experiment was conducted on 4-weeks mice which had caloric restrictions for 9 months. It was observed that malnourished mice had bigger amount of VSEL and HSPC (stem cells and progenitor haematopoietic cells) in comparison with control group. It was stated that diet with limited calories can positively influence on VSEL pool thereby on extension of animals life [14,15].

The results of described researches differentiate from this presented by Lazare S. et al. (2017) [16]. In this study the influence of long-standing caloric restrictions and diet with too much calories on the number and functions of HSC were analysed. The results were compared with control group. It turned up that regardless of used diet in all experimental groups constant increase of HSC caused by ageing of animals was noted. The improvement of HSC functioning after caloric restrictions was not observed. This inconsistent to previous researches results might be caused by differences in experimental project. However to exclude the influence of outside factors more studies should be carried out.

\section{The influence of protein-caloric malnutrition on haematopoiesis process}

The protein malnutrition is the state which can lead not only to deficit of all morphotic blood elements (pancytopenia) and leukopenia but also to inhibition of haematopoiesis. In Cunha et al. (2013) research two-month male mice were under observation with $2 \%$ of protein content diet [6]. The diet of control group (ab libitum) included $12 \%$ of protein. The experiment lasted 21 days. In this time animals qualified to the research lost about $20 \%$ of body weight. Haematopoietic parameters, CD45, CD117 were analysed as the differentiation cell antigens recognized as early marker of haematopoietic stem cells. The results of conducted research indicate the appearance of anaemia and leukopenia in malnourished mice. There was also decrease of expression of CD45 and CD117 cells and hypoplasia of bone marrow leading to pancytopenia caused by bone marrow impairment.
The CD45 protein impact on the regulation of different states of lymphocytes maturation. The role of this protein in the process of basic unmatured haematopoietic cells was showed [11,22].

There are a lot of researches analysing the influence of protein-caloric malnutrition on the haematopoietic processes and the proliferation activity of haematopoietic cells. In these studies after the usage of appropriate amount of protein (4-5\%) and losing about $20-25 \%$ of weight some phenomena were observed: decreased bone marrow volume, hypoplasia, stopping of maturation of the cells, leukopenia and lymphopenia with decreased number of lymphoid cells line. The inflammatory response to LPS (lipopolysaccharide which is bacterial endotoxin) was tested in experiment carried out by Fock et al. (2007) [8]. LPS is the stimulus to forming of TNF- $\propto$ and IL-1, proinflammatory cytokines which stimulate humoral and cellular response, proliferation of lymphocytes and neutrophil aggregation and also IL-6, cytokine which activates bone marrow haematopoiesis. The results indicate decrease of cytokine production (TNF- $\propto$, IL-1, IL-6) what is indicative of limited ability to immune response in malnourished animals. In Borelli et al. (2009) research attention was paid on cell cycle phases in which bone marrow cells are and the percentage of progenitor cells in bone marrow [4]. Malnourished animals in comparison with control group had more cells in G0 phase of cell cycle as the results show. To sum up, it has been stated that protein-caloric malnutrition might lead to depletion of haematopoietic cells and also to developmental changes.

\section{Summary}

There were many reports published referring to changes in peripheral blood in animals with limited feed. However, only some of them were focused on the influence of malnutrition on haematopoietic and erythroid cells in bone marrow. In view of wide interest of animal model in assessment of safety i.e. drugs, exploration of the topic and conducting additional researches should be considered. Results of these researches would help toxicologists to recognise secondary changes caused by food limitation and to focus on primary consequences of drug activity. It should be noted that high doses of medicines often cause reluctance to food what might influence on nutritional status of animals. Results of 
researches carried out on rats which had limited feed and took additionally 5-FU also suggest caution in analysing hematotoxicity drugs tests. The focus should be on finding the best dose $(\%)$ of feed in order to minimise the influence of malnutrition on toxicological effect of medicine.

Caloric restrictions in the level which does not bring malnutrition might have in turn positive impact on organism in terms of ageing and preventing of chronic diseases. More researches analysing long-term effects of moderate limitation of food on ability of HSC to repopulation and maintenance of lymphocytopoesis should be carried out in view of observed at the same time negative consequences.

Haematopoiesis is the process that consists in the proliferation and differentiation of bone marrow stem cells leading to forming of mature cells of bone marrow and releasing them to bloodstream. This process is vulnerable to requirements of specific cell line. This need high availability of diet components including proteins to proper process. Overall malnutrition and deficit of protein might cause hypoplasia and decrease of ability to produce lymphocytes, disturbing organism immunity.

Another very little known phenomenon is a diet composition and its influence on morphological construction of bone marrow and peripheral blood. As other researches showed the kind of diet (high fat, high carbohydrate, high cholesterol and high fructose) significantly impact on biochemical parameters of blood. There is a lack of works concerning this issue so far. There are such works as Krośniak et al. (2013) which tell about possible influence of diet composition with no restrictions on peripheral blood and construction of bone marrow [13]. Conclusions of this researches indicate that the kind of diet makes an important contribution to blood and bone marrow. The emphasis is put by researchers on high fat diet in terms of changes on stem cell level (HSC) and progenitor (HSPC) in bone marrow [25]. This diet inducing obesity conduces to decrease of population of HSC and HSPC and cause leucocytosis and neutrophilia. The influence of other kinds of diet on morphological construction of bone marrow is little known. This is the chance for researchers to carry out subsequent studies and broaden knowledge in this area.

Conflict of Interest: The authors declare no conflict of interests.

\section{References}

1. Anagnostou A, Schade S, Ashkinaz M, Barone J, Fried W. Effect of protein deprivation on erythropoiesis. Blood. 1977;50:1093-1097.
2. Asanuma F, Miyata H, Iwaki $Y$, Kimura M, Matsumoto K. Evaluation of Short-term Myelotoxicity Study in Dietary Reduced Rats. J Toxicol Pathol. 2010;23: 31-37.

3. Asanuma $F$, Miyata $H$, Iwaki $Y$, Kimura $M$, Matsumoto K. Importance of starting age for myelotox- icity study in dietary restricted rats. J Toxicol Pathol. 2009;22:153-166.

4. Borelli P. et al. Protein-energy malnutrition halts hemopoietic progenitor cells in the G0/G1 cell cycle stage, thereby altering cell production rates. Braz J Med Biol Res. 2009;42:523-530.

5. Catchatourian R, Eckerling G, Fried W. Effect of short-term protein deprivation on hemopoietic functions of healthy volunteers. Blood. 1980; 55:625-628.

6. Cunha M.C, et al. Protein malnutrition induces bone marrow mesenchymal stem cells commitment to adipogenic differentiation leading to hematopoietic failure. PLoS One. 2013;8:e58872.

7. Dunn C.D. Effect of food or water restriction on erythropoiesis in mice: relevance to "anemia" of space flight. Am J Physiol. 1980;238:301-5.

8. Fock R.A, Vinolo M.A, de Moura Sá Rocha V, de Sá Rocha L.C, Borelli P. Protein-energy malnutrition decreases the expression of TLR4/MD-2 and CD14 receptors in peritoneal macrophages and reduces the synthesis of TNFalpha in response to lipopolysaccharide (LPS) in mice. Cytokine. 2007;40:105-114.

9. Fontana L., Partridge L., Longo V.D. Extending healthy life span-from yeast to humans. Science. 2010;328:321-326.

10. Gurion R, et al. Hematopoetic growth factors in aplastic anemia patients treated with immunosuppressive therapy-systematic review and meta-analysis. Haematologica. 2009;94:712719.

11. Hermiston M.L, Tan A.L, Gupta V.A, Majeti R, Weiss A. The juxtamembrane wedge negatively regulates CD45 function in B cells. Immunity. 2005;23:635-647.

12. Hishinuma K., Kimura S. Dietary restriction augments erythropoiesis in mice. Int $\mathbf{J}$ Vitam Nutr Res. 1990;60:379-82.

13. Krośniak $M$, et al. Influence of Fructose and Fatty-Rich Diet Combined with Vanadium on Bone Marrow Cells. Biol Trace Elem Res. 2013; 155:276-282. 
14. Kucia $M$, et al. The negative effect of prolonged somatotrophic/insulin signaling on an adult bone marrow-residing population of pluripotent very small embryonic-like stem cells (VSELs). Age (Dordr). 2013; 35:315-330.

15. Kucia M, et al. A population of very small embryoniclike (VSEL) CXCR4(+) SSEA-1(+) Oct-4+ stem cells identified in adult bone marrow. Leukemia. 2006;20:85769.

16. Lazare $S$, et al. Lifelong dietary intervention does not affect hematopoietic stem cell function. Exp Hematol. 2017;53:26-30.

17. Levin S, Semler D, Ruben Z. Effects of Two Weeks of Feed Restriction on Some Common Toxicologic Parameters in Sprague-Dawley Rats. Toxicol Pathol. 1993;21:1-14.

18. Mantel C, et al. Mouse hematopoietic cell-targeted STST3 deletion: stem/progenitor cell defects, mitochondrial dysfunction, ROS overproduction and a rapid aging-like phenotyp. Blood. 2012;120:2589-2599.

19. Moriyama $T$, et al. Beneficjal effect of moderate food restriction in toxicity studies in rats. J Toxicol Sci. 2006;3:197-206.
20. Moriyama T, et al. Effects of reduced food intake on toxicity study parameters in rats. $\mathrm{J}$ Toxicol Sci. 2008;33:537-47.

21. Pearse G. Histopathology of the thymus. Toxicol Pathol. 2006;34:515-547.

22. Shivtiel S, et al. CD45 regulates retention, motility, and numbers of hematopoietic progenitors, and affects osteoclast remodeling of metaphyseal trabecules. J Exp Med. 2008;205:2381-2395.

23. Tang $D$, et al. Dietary restriction improves repopulation but impairs lymphoid differentiation capacity of hematopoietic stem cells in early aging. J Exp Med. 2016;213:535553.

24. Tian L, Yu X. Fat, Sugar, and Bone Health: A Complex Relationship. Nutrients. 2017;9:506.

25. Van den Berg S.M, et al. Diet-induced obesity in mice diminishes hematopoietic stem and progenitor cells in the bone marrow. FASEB J Off Publ Fed Am Soc Exp Biol.2016;30:17791788. 